Revue de l'association française de recherche sur

I'histoire du cinéma

$91 \mid 2020$

Ivresse patrimoniale ? - Décors d'Intolérance -

Germaine Dulac - Alice au pays des merveilles - Éric

Rondepierre

\title{
Andrei Tarkovsky The Exhibition, Eye Filmmuseum / Free Flight, Nouvelle Galerie Tretiakov
}

Loïc Millot

\section{OpenEdition}

\section{Journals}

Édition électronique

URL : https://journals.openedition.org/1895/8058

DOI : 10.4000/1895.8058

ISSN : $1960-6176$

Éditeur

Association française de recherche sur l'histoire du cinéma (AFRHC)

Édition imprimée

Date de publication : 1 juin 2020

Pagination : 169-171

ISBN : 978-2-37029-091-5

ISSN : 0769-0959

Référence électronique

Loïc Millot, «Andrei Tarkovsky The Exhibition, Eye Filmmuseum / Free Flight, Nouvelle Galerie

Tretiakov », 1895. Mille huit cent quatre-vingt-quinze [En ligne], 91 | 2020, mis en ligne le 10 mai 2021 consulté le 01 juin 2021. URL : http://journals.openedition.org/1895/8058 ; DOI : https://doi.org/ $10.4000 / 1895.8058$

Ce document a été généré automatiquement le 1 juin 2021

(c) $\mathrm{AFRHC}$ 


\title{
Andrei Tarkovsky The Exhibition, Eye Filmmuseum / Free Flight, Nouvelle Galerie Tretiakov
}

\author{
Loïc Millot
}

1 Du 14 septembre au 6 décembre 2019 s'est tenue au Eye Filmmuseum, Andrei Tarkovsky The Exhibition, la première manifestation importante qui soit consacrée en Hollande à l'œuvre du cinéaste soviétique. Par un hasard du calendrier, celle-ci débutait au moment même où s'achevait, à Moscou, un projet qui rendait hommage au poète visionnaire d'une autre manière. Deux démarches curatoriales étaient ainsi proposées au public en l'espace de quelques mois. À Amsterdam, l'exposition monographique, conçue par Jaap Guldemond et Marente Bloemheuvel, était centrée sur les films de l'auteur, en s'appuyant sur une sélection de fragments exaltant la dimension poétique et spirituelle de son œuvre. À la Nouvelle Galerie Tretiakov, où prenait place l'installation Free Flight (4 juin-22 septembre 2019), les longs-métrages d'Andreï Tarkovski côtoyaient les œuvres de plasticiens soviétiques réunis sous la bannière du «non-conformisme » et de l'art «non-officiel» - un aspect de l'histoire des arts qui connaît ces dernières années un regain d'intérêt en France (Cf. Kollektsia! Art contemporain en URSS et en Russie. 1950-2000, du 14 septembre-2 avril 2017 au Centre Pompidou; Les non-conformistes. Histoire d'une collection russe, du 13 novembre au 9 février 2020 au MO.CO. Hôtel des collections de Montpellier). Il s'agissait, de façon stimulante et audacieuse, de revisiter l'œuvre de Tarkovski à l'aune de ces artistes qui ont exercé à la même époque (1960-1980), depuis le bref « Dégel » initié dans les arts par Khrouchtchev jusqu'à l'ère brejnevienne. La collaboration entre la Galerie Tretiakov et le musée AZ (pour l'artiste Anatoli Zverev, 1931-1986) fondé en 2015 par Natalia Opaleva et Polina Lobatchesvkaïa prenait ici tout son sens, puisque ce sont des collections de ce musée privé que provenaient les soixante-dix pièces (tableaux, esquisses, sculptures) réunies aux côtés des œuvres du cinéaste. Contrairement à l'exposition d'Amsterdam, qui rassemblait l'intégralité des films en plus de leur distribution nationale en salles, seuls trois longs métrages de la période soviétique étaient convoqués à Moscou : Andreï Roublev (1966-1971), Solaris (1972) et Stalker (1979). 
Trois genres (la fresque religieuse ; la science-fiction ; la dystopie) autour desquels s'est construite l'installation Free Flight, qui en déclinait les univers sur toute l'aile ouest de la Nouvelle Galerie Tretiakov. Ainsi, une première section rapprochait des extraits d'Andrei Roublev des toiles méditatives de Dmitri Plavinski (1937-2012), l'un des principaux représentants du mouvement non-conformiste en Russie. Plus loin, les vestiges industriels de la Zone (Stalker) rencontraient les toiles de Piotr Belenok (1938-1991) et les vues photographiques de la centrale de Tchernobyl prises en 1990 par la journaliste Victoria Ivleva, lauréate du World Press Photo. Outre les dessins et tableaux des représentants de l'art non-conformiste (Anatoli Zverev, Francisco Infante, Ülo Sooster...), une troisième section accueillait une architecture monumentale inspirée des décors de Mikhaill Romadine pour le vaisseau spatial de Solaris.

2 Cette forme spectaculaire contrastait avec celle, résolument sobre et intimiste, voulue par Jaap Guldemond pour Andrei Tarkovsky The Exhibition. Nimbée dans une sousexposition de rigueur - des conditions de luminosité qui semblent se généraliser parmi les institutions muséales -, l'atmosphère d'ensemble était prompte à accueillir les méditations spirituelles du cinéaste croyant. Resserrée sur les longs métrages, et donc à l'exclusion des courts réalisés dans le cadre de la formation de Tarkovski au VGIK, l'exposition présentait des extraits sur un, deux ou trois écrans contigus, tel un gigantesque retable constitué de fugues et de fulgurances visuelles. Ainsi qu'est présentée la manifestation sur le site du Eye Filmmuseum, l'intention des curateurs était de coller au plus près des films et des considérations esthétiques développées par l'auteur du Temps scellé (Éditions de l'Étoile / Cahiers du cinéma, 1989). Le montage et le choix des extraits visait ainsi à produire des associations poétiques chères à Tarkovski ; de même que chacun de ses films aspire in fine à restaurer le culte orthodoxe mis à mal par le régime soviétique, tout l'espace d'exposition du Eye Filmmuseum adoptait la solennité recueillie d'une église. On peut cependant regretter de la part d'une institution chargée de promouvoir l'interdisciplinarité que les autres fonctions et activités de Tarkovski n'aient nullement été évoquées. Elles sont pourtant nombreuses et encore peu connues du grand public, de la musique à la peinture dans sa jeunesse, en passant par ses velléités littéraires sous l'influence de son père et ses mises en scène pour le théâtre et l'opéra. Cette ouverture disciplinaire, c'est dans l'intimité des images qu'il fallait la rechercher : en prenant part au culte de la parturition rendu à la Madonna del Parto (dans Nostalghia), en s'inclinant devant l'enfant muet sur le modèle de l'Adoration des mages (dans le Sacrifice), en écoutant un poème lu par Arseni Tarkovski (dans le Miroir), ou en s'élevant dans un tourbillon d'icônes et de chants liturgiques au terme d'Andreï Roublev, lorsque le film devenant iconostase finit par révéler l'entreprise cultuelle du cinéaste. À l'instar de Sergueï Mikhaïlovitch Eisenstein et de Pier Paolo Pasolini, chaque long métrage de Tarkovski s'ancre dans une histoire générale des cultes et des arts.

Cette expérience sensorielle, extatique, était ponctuée d'archives privées mises à disposition par le fils cadet du cinéaste, Andreï Andreïevitch Tarkovski : photographies de tournage, scénarios de longs métrages, lettres de collègues (italiens notamment: Michelangelo Antonioni, Tonino Guerra). Lorsqu'ils ne se chevauchaient pas en une disposition illisible, les documents non traduits et accompagnés de légendes bien trop succinctes étaient indéchiffrables pour un public étranger à la langue russe. On regrette d'autant plus cette lacune que figuraient de précieux rapports de la commission de censure, parmi lesquels celui incriminant les séquences de violence et de nudité 
païenne dans Andreï Roublev. La valorisation de la matière filmique se réalisait ainsi au détriment de son intelligence contextuelle. En faisant l'impasse sur l'idéologie politique de Tarkovski ou sur son rapport à la religion orthodoxe, dont il ne cesse pourtant de promouvoir la ferveur et le messianisme, le public demeure captif de la beauté éblouissante des images. C'est nier, en outre, que les formes cinématographiques résultent elles aussi d'un rapport de force avec les autorités soviétiques, comme en témoignent les nombreux détours qu'emprunte le langage poétique de Tarkovski.

4 L'autre ouverture proposée par le Eye Filmmuseum reposait sur la série de polaroids réalisée au moment où le cinéaste était occupé au tournage de Nostalghia, entre Moscou et la Toscane. Bien connue des adorateurs de son œuvre, puisqu'elle a fait l'objet de nombreuses publications éditoriales, cette série photographique était montrée aux Pays-Bas pour la première fois. Présenté en introduction de l'exposition, l'ensemble est imprégné de l'atmosphère vaporeuse qui enveloppe ses films. Derrière chaque appareil de prises de vue, l'œil est en effet le même. On y retrouve les compositions "impressionnistes", un traitement similaire des couleurs ainsi que les motifs récurrents de ses longs métrages en couleurs : les clartés automnales dans le Miroir, la brume matinale des thermes de Bagno Vignoni (dans Nostalghia), ou encore l'arbre esseulé au milieu d'un pré, figure qui sera posée au principe et à la fin du Sacrifice. En marge de la manifestation, un programme proposait une sélection de films des quelques maîtres que reconnaissait le cinéaste: Alexandre Dovjenko (la Terre, 1930), Charlie Chaplin (les Lumières de la ville, 1931), Robert Bresson (Journal d'un curé de campagne, 1951), Kenji Mizoguchi (les Contes de la lune vague après la pluie, 1953), Luis Buñuel (Nazarin, 1958), Sergueï Paradjanov (la Couleur de la grenade, 1969), Ingmar Bergman (la Honte, 1968). À ce panthéon de l'auteur étaient ajoutés des cinéastes qui lui ont rendu hommage (Chris Marker) ou qui en poursuivent aujourd'hui l'esthétique de la durée - Lars von Trier (Melancholia, 2011), Carlos Reygadas (Japón, 2002), Andreï Zviaguintsev (le Retour, 2003). Figurait aussi le spectacle Nostalgia for the Absolute (2019) mis en scène par Andreï Andreïevitch Tarkovski, président de l'Institut international Andreï Tarkovski et auquel, on s'en souvient, fut dédié le Sacrifice.

5 Là où l'exposition d'Amsterdam cédait à la fétichisation des films en les séparant de leur contexte politique, l'installation Free Flight semblait restaurer ce lien en rassemblant des artistes dont les carrières se sont construites en rapport avec la censure d'État. La contestation du réalisme socialiste contraignait à exercer en marge de la société, à produire des œuvres cantonnées à des apparitions ponctuelles voire clandestines : dans des caves ou lors d'expositions éphémères pour les plasticiens, au sein de réseaux de ciné-clubs pour les cinéastes. Les difficultés professionnelles rencontrées par Tarkovski en URSS peuvent nous rappeler les écueils auxquels furent confrontés les plasticiens depuis l'exposition Nouvelle Réalité organisée en 1962 au Manège (Moscou). L'intention qui présidait à ce rapprochement était donc séduisante, et sans doute répondait-elle à l'attrait qu'exercent en art toutes sortes de transgression - dont la récente « affaire » Pavlenski aura montré les limites. Le rapport à la censure était surtout prétexte à célébrer une période d'intense créativité dans les arts (1960-1980) qualifiée, rétrospectivement, de "Renaissance soviétique ». Ce qui a pour effet paradoxal de venir justifier la censure d'État, dès lors que celle-ci a positivement contribué à produire un art "non-officiel ». À placer les artistes du seul côté de l'art non-officiel, on fige en outre une relation avec les autorités soviétiques qui est susceptible d'évoluer dans le temps et peut donc se prêter à réversibilité. La frontière entre art officiel et non-officiel est poreuse selon les circonstances. Loin d'être un 
artiste maudit ou l'unique bouc-émissaire du régime soviétique, une image largement répandue en Europe et entretenue par le cinéaste lui-même (en nommant par exemple son journal «martyrologe»), Tarkovski occupe une position ambivalente dans le cinéma soviétique. Malgré ses démêlés avec la censure, il obtint des budgets importants pour le tournage de ses films (Andrë Roublev), reçut de l'État une commande ambitieuse (Solaris), fut promu Artiste du peuple et dispensait des cours au VGIK. Plus qu'aucun autre cinéaste soviétique à cette époque, il bénéficia très tôt de la bienveillance et de l'appui diplomatique des plus grands festivals européens (Cannes, Venise).

6 Malgré ces simplifications, l'installation à la Nouvelle Galerie Tretiakov avait le mérite de proposer des rapprochements suggestifs et productifs sur le plan formel, et d'instaurer une dynamique d'expérimentation curatoriale interdisciplinaire inédite pour ce lieu. 